\title{
The SuperNEMO Demonstrator calorimeter commissioning
}

\author{
Malak HOBALLAH ${ }^{a, *}$ \\ ${ }^{a}$ IJCLab, Université Paris-Sud, CNRS/IN2P3, Université Paris-Saclay, \\ 15 Rue Georges Clemenceau, 91400 Orsay, France \\ E-mail: hoballah@lal.in2p3.fr
}

The SuperNEMO experiment aims to search for $0 v \beta \beta$ : this discovery would prove the Majorana nature of neutrinos. The final detector goal is to reach a sensitivity of $10^{26}$ years on the $0 v \beta \beta$ decay half-life, i.e an effective Majorana neutrino mass of $50-100 \mathrm{meV}$. The first module of the SuperNEMO experiment is under installation at Laboratoire Souterrain de Modane (LSM), with $6.23 \mathrm{~kg}$ of ${ }^{82} \mathrm{Se}$. Electrons from disintegrations are tracked when crossing the wire chamber, then their energies and arrival times are measured by the calorimeter. The calorimeter is composed of 712 optical modules, an assembly of large volume plastic scintillators couples to PMTs. The commissioning of the SuperNEMO calorimeter began in 2019. An optimised parameterisation of the PMT signal waveforms has been developed. The calorimeter response has been calibrated in energy using the internal ${ }^{208} \mathrm{Tl}$ spectrum, and photomultipliers' gains were equalised at $1 \mathrm{MeV}$ with $<10 \%$ spread over all the calorimeter walls. $\mathrm{A}^{60} \mathrm{Co}$ source, emitting two gammas simultaneously, was used to generate preliminary measurements of the time resolution of all optical modules of $<600$ ps at $1 \mathrm{MeV}$.

40th International Conference on High Energy physics - ICHEP2020

July 28 - August 6, 2020

Prague, Czech Republic (virtual meeting)

\footnotetext{
${ }^{*}$ Speaker
} 


\section{Introduction}

SuperNEMO is an experiment that inherits its instrumentation and principle from its predecessor, NEMO-3. experiment, it will search for the neutrinoless double beta $(0 v \beta \beta)$ decay using a tracker-calorimeter technique. This technique allows a full topological reconstruction of particles, and a measurement of the individual particles' energies and times-of-flight, which allows a unique identification of the different backgrounds to be able to reach an ultra-low background count. With such background rejection and technique, we should be able to study the different decay mechanisms of two neutrino double beta $(2 v \beta \beta)$ decay and, if it exists, the $0 v \beta \beta$ decay mechanism(s) by reconstructing the full kinemetics (single electron energy and angular distribution) of the $\beta \beta$ events.

A first module of SuperNEMO, called Demonstrator, is under construction and testing at the Laboratoire Souterrain de Modane (LSM) at 4800 m.w.e. depth. The Demonstrator aims to reach a sensitivity of $T_{1 / 2}^{0 v}>6.5 \times 10^{24}$ y corresponding to $\left\langle m_{v}\right\rangle<(260-500) \mathrm{meV}$ with $17.5 \mathrm{~kg} . \mathrm{y}$ exposure of ${ }^{82} \mathrm{Se}$. With this Demonstrator, we can also prove that a SuperNEMO module can reach the background specifications for source radio-purity : ${ }^{214} \mathrm{Bi}<2 \mu \mathrm{Bq} / \mathrm{kg}$ and ${ }^{208} \mathrm{Tl}<10 \mu \mathrm{Bq} / \mathrm{kg}$ and Radon activity : ${ }^{222} \mathrm{Rn}<0.15 \mathrm{mBq} / \mathrm{m}^{3}$ ).

\section{The SuperNEMO Demonstrator}

The SuperNEMO Demonstrator is composed of three main components: the source foils, the tracker and the calorimeter (see Figure 1). The source foils have been designed with $6.23 \mathrm{~kg}$ of ${ }^{82} \mathrm{Se}$. This isotope has a $Q_{\beta \beta}=2.99 \mathrm{MeV}$, an energy region where the natural $\beta / \gamma$ environmental background is reduced. Its relatively high $2 v \beta \beta$ half-life of $T_{1 / 2}^{2 v}=9.4 \times 10^{19}$ will decrease the $2 v \beta \beta$ background compared to the previous NEMO-3 experiment (which uses mainly ${ }^{100} \mathrm{Mo}$ ), with respect to $0 v \beta \beta$ events. The source module is sandwiched by two wire tracking chamber with 2034 drift cells operating in Geiger mode and filled with a gas mixture of $95 \% \mathrm{He}, 4 \%$ ethanol and $1 \%$ Ar. The tracker surrounds the source foils in order to have a full topological reconstruction of the $\beta \beta$ events and to have high background rejection. This whole setup is enveloped with a calorimeter of 712 optical modules (OMs), of which 440 are 8" OMs having an energy resolution of 8\% FWHM at $1 \mathrm{MeV}$, and a time resolution of $\sigma<400$ ps for $1 \mathrm{MeV}$ electrons. A coil producing a $25 \mathrm{G}$ magnetic field will be installed to distinguish different particles charges and directions of travel, and a anti-radon tent to prevent Radon from diffusing from the laboratory towards the tracker. An iron shield of $20 \mathrm{~cm}$ thickness to protect against gamma rays from the laboratory and a second shield consisting of polyethylene water tanks and boron polyethylene plates to thermalize and capture neutrons emitted outside the detector, will also be installed.

\section{The SuperNEMO Calorimeter Commissioning}

The SuperNEMO calorimeter is being commisioned and is taking data since 2019. Runs, tests and analysis were made to check its functioning.

\subsection{Energy Calibration and Photomultipliers High-Voltage Gain Calibration}

Since the Demonstrator tracker has not yet been commisioned, the photomultipliers' (PMTs) high voltage gain was equalized using the Compton edge of the $2.614 \mathrm{MeV} \gamma \mathrm{s}$ from the ${ }^{208} \mathrm{Tl}$ 


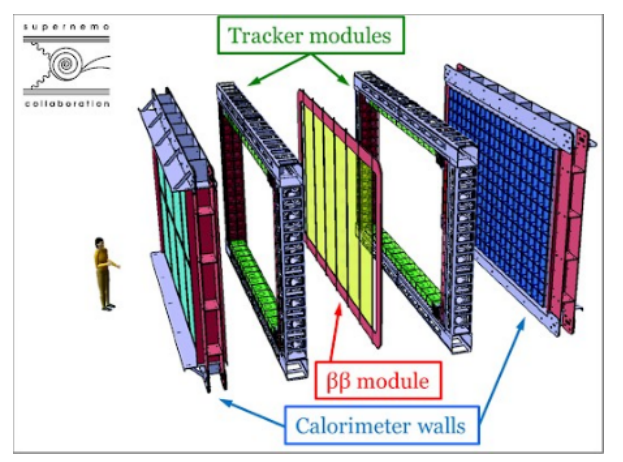

Figure 1: A sketch of the SuperNEMO Demonstrator showing the source foils position, the tracker and the calorimeter walls.

background. Using this equalization procedure, we were able to reduce the spread of the OMs' gain to less than $10 \%$. A better precision is expected using an electron emitting source $\left({ }^{207} \mathrm{Bi}\right)$ and the tracker running.

\subsection{Baseline Calculations}

Since our PMT signals are digitized by electronic boards, baseline calculations were made to ensure the stability of our electronics. The OMs' mean baselines were measured for equal time periods over multiple runs of data taking, confirming that our electronics were very stable, with low noise.

\subsection{Reflectometry tests}

Reflectometry tests were made: a primary signal is sent from the electronics boards to the OMs, where it is reflected back. This kind of test allows us to check the correct operation of the calorimeter. First, it allows us to check the cable connections at the patch panel and OM ends. Second, we can measure the signal attentuation created by the cables from studying the reflected signal shape. The time delay between the initial and reflected pulses, proportional to the cable length between each OM and the electronics boards, will be used for timing calibration.

\subsection{Preliminary Time Resolution Results}

Preliminary analysis for the time resolution was carried out using a ${ }^{60} \mathrm{Co}$ source which emits two $\gamma \mathrm{s}$ simultaneously, with energies $1.17 \mathrm{MeV}$ and $1.33 \mathrm{MeV}$. This ${ }^{60} \mathrm{Co}$ source was placed at different positions behind the Demonstrator calorimeter wall, we take data runs and study two signals in two OMs detected in coincidence inside a certain time window, and then study the time difference between them and perform fits. After comparing with simulations, a preliminary time resolution result is found to be $<600 \mathrm{ps}$ for $\gamma \mathrm{s}$ at $1 \mathrm{MeV}$. Much better results are expected with an electron source $\left({ }^{207} \mathrm{Bi}\right)$ and tracker running.

\section{References}

[1] F.Mauger, Conseil Scientifque IN2P3 Expérience SuperNEMO, 2018. 